\title{
MOPP-ABV Regimen
}

National Cancer Institute

\section{Source}

National Cancer Institute. MOPP-ABV Regimen. NCI Thesaurus. Code C9620.

A chemotherapy regimen consisting of sequential administration of mechlorethamine, vincristine, procarbazine, and prednisone (MOPP) followed by doxorubicin, bleomycin, and vinblastine (ABV), that may be used in the treatment of Hodgkin lymphoma $(\mathrm{HL})$. 